\title{
Successful Treatment of Foreign Body Granuloma Using Topical Photodynamic Therapy
}

Jin Young Kim

Eun Soo Park

Department of Plastic and Reconstructive Surgery, Soonchunhyang Univsersity Bucheon Hospital, Bucheon, Korea
Received May 30, 2014

Revised June 21, 2014

Accepted June 23, 2014

\section{Correspondence}

Eun Soo Park

Department of Plastic and Reconstructive Surgery, Soonchunhyang Univsersity Bucheon Hospital, 1174, Jung-dong, Wonmi-gu, Bucheon 420-767, Korea

Tel: +82-32-621-5319

Fax: +82-32-621-5016

E-mail: peunsoodschmc.ac.kr

(C) Korean Society for Laser Medicine and Surgery

(c) This is an open access article distributed under the terms of the Creative Commons Attribution NonCommercial License (http://creativecommons.org/ licenses/by-nc/3.0) which permits unrestricted noncommercial use, distribution, and reproduction in any medium, provided the original work is properly cited.
Recently, filler injection has been widely used in treatment of wrinkles of adult women or to boost up the forehead, eyebrows, lips, cheeks area, etc. It is a relatively less invasive procedure requiring only a simple process and rapid return to normal life, which is growing in popularity. However, its side effects have also increased. The most serious complications of the delayed side effects include granuloma formation by reaction to an alien substance. The frequency of alien substance-related granuloma is reported to be lower than that of more recently developed filler injection methods, however, its ratio is estimated to be up to approximately $3 \%$ as filler-using plastic surgeries increase and are performed by large numbers of non-medical experts using unknown ingredients in unauthorized clinics in South Korea. Granuloma formation by reaction to an alien substance is a normal tissue reaction that appears to stop any material that is not immediately removed by enzyme destruction or phagocytosis from moving elsewhere and isolate it. We report on a case of granuloma formation after filler injection treatment using the $660 \mathrm{~nm}$ bio-light and Chlorophyll-lipoid complex (CLC, VIRTAHealer $^{T M}$ ] as a photosensitizer. The photodynamic therapy is a phototreatment involving absorption of a photosensitizer, a material that reacts to light, into a target cell, which uses flashing light in a specific wave length in order to selectively destroy targeted lesions. The $660 \mathrm{~mm}$ biolight stimulates cells involved in cut wound treatment and facilitates blood circulation to promote skin re-generation.

\section{Key words}

Filler; Foreign body granuloma; Photodynamic therapy 


\section{INTRODUCTION}

For the beauty of women, facial contour procedures using diverse materials have been performed from long ago. Recently, in order to treat wrinkles of adult women or to boost up forehead, eyebrows, lips, cheeks area, etc. filler injection has been widely operated. 'It is a relatively less invasive procedure requiring only simple process and fast return to normal life, gaining growing popularity. However, its side effects also increase. Usually, depending on the point of occurrence, they are divided into acute and delayed side effects. The most serious cases of the acute side effects include over excessive filler injection, dermal necrosis due to intra-vessel injection-caused embolism, blindness, etc. And the most serious complications of the delayed side effects include foreign body granuloma formation by reaction to alien substance. ${ }^{2}$ The frequency of foreign body granuloma is reported to be lower regarding more recently developed filler injection method but its ratio is estimated to be approximately up to $3 \%$ as filler-using plastic surgeries increase and still lots of non medical experts perform it with unknown ingredients in unauthorized clinics. ${ }^{3}$ The foreign body granuloma formation by reaction to alien substance is a normal tissue reaction that seems to stop any material failed to be immediately removed by enzyme destruction or phagocytosis from moving elsewhere and isolate it. However, as expert practitioners use fillers with less impurities (Artecoll ${ }^{\circledR}$ ), etc. than existing fillers, their granuloma formation by reaction to alien substance is being decreased from $0.1 \%$ to $0.01 \%$. ${ }^{2,3}$

\section{CASE REPORT}

This case is on a 56-year-old female patient who has received filler injection procedures with unknown ingredients in her nose, forehead, and both nasolabial areas about 11 years ago. She received a diagnosis
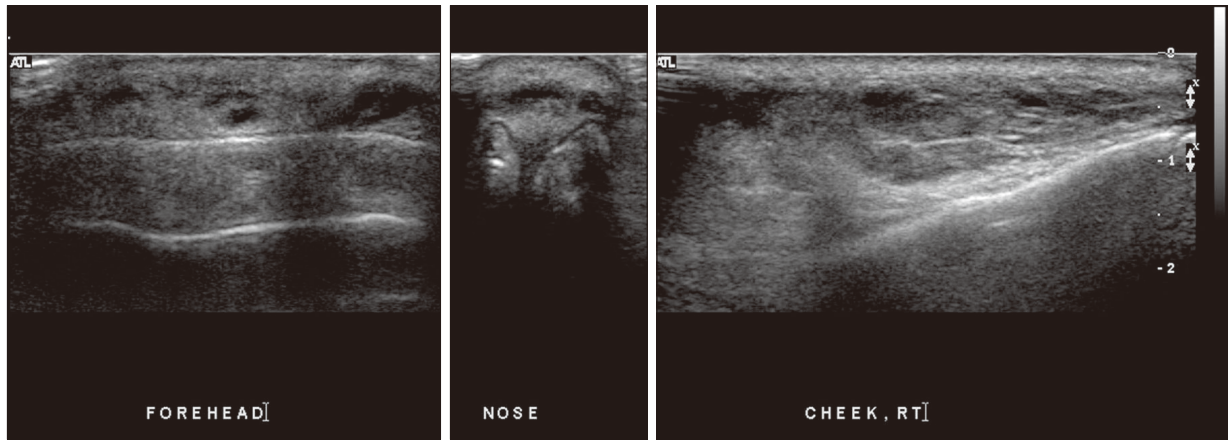

Fig. 1. Ultrasonography image findings: The margin of granuloma is unclear; ground glass appearance.
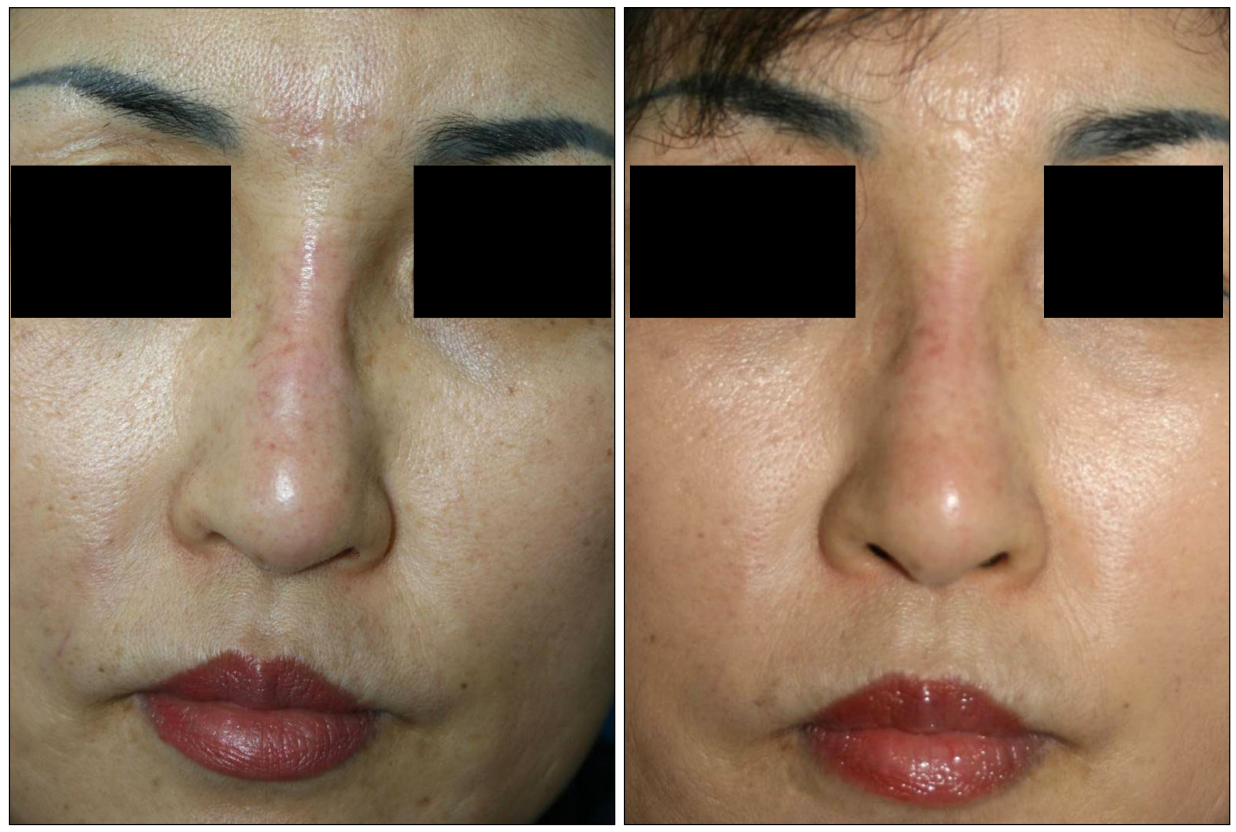

Fig. 2. (Left) Photofinding: Hemotelangiosis on nasal dorsum. (Right) Photofinding: After two times of VersaPulse (long-pulse $532 \mathrm{~nm}$ ) vascular laser-based treatment. 

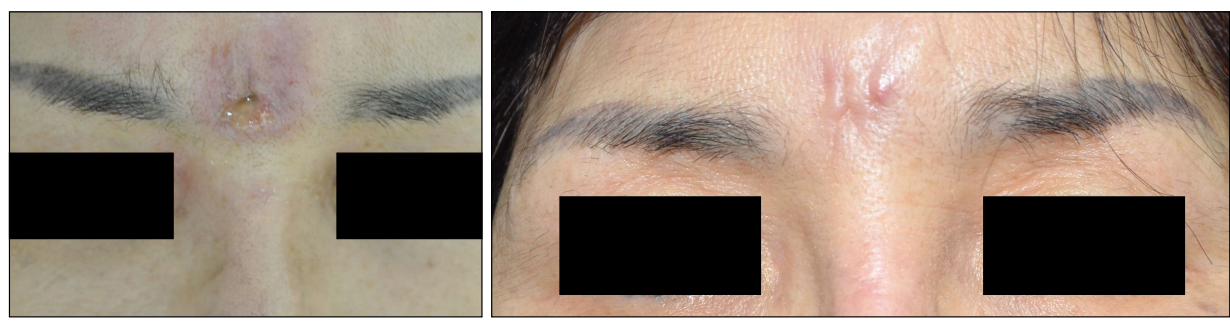

Fig. 3. (Left) Initial photofinding: Ulceration on face after filler injection. (Right) Post-procedure photofinding.

of foreign body granuloma formation by reaction to alien substance about 39 months ago when she came to receive an ultrasonography for her facial multiple nodules, heat sensation, pressure ache, and nasal area hemotelangiosis, foreign body granuloma was seen with unclear demarcation and no capillary increase (Fig. 1). Triamcinolone acetonide $40 \mathrm{mg} / 1 \mathrm{ml}$ was administered and two times of VersaPulse (long-pulse $532 \mathrm{~nm}$ ) vascular laser-based treatment were conducted for the nasal dorsum hemotelangiosis back then and she was improved (Fig. 2). The patient visited the hospital again in 3 years for ulcerative nodes between her eyebrows (Fig. 3 Left). In terms of physical science, a $2 \times 1.5 \mathrm{~cm}$-sized node was observed with serous secretion which was fixed between the eyebrows with clearer demarcation. No other special condition was found other than the lesion area. Local steroid injection and antibiotics for the whole body was used. No identified strain was observed in the germiculture examination. Excochleation was conducted around the ulcer. Then, from the $7^{\text {th }}$ day of her first hospital visit, for every 2-3 days, Chlorophylllipoid complex (CLC, VIRTA-Healer ${ }^{T M}$ ) was spread as a photosensitizer for 20 minutes and removed. $660 \mathrm{~nm}$ Bio-light was shed for 20 minutes for 4 times. After the 4 rounds of Bio-light flashing, the size of the cut wound decreased to about $4 \times 4 \mathrm{~mm}$ and healthy red granulation tissue growth was observed at the bottom (Fig. 3 Right). In about 10 days, it was completely treated.

\section{DISCUSSION}

In most of the cases, normal side effects after filler injection such as bruise, edema, pain, skin discoloration, etc. are minor and recover within 3 days. ${ }^{4}$ But in some cases, skin becomes uneven as lumps or develops hematoma, granuloma, etc. to cause a problem in the appearances. The mechanism or process of postfiller injection granuloma formation, in particular, has not been clearly reported so far. But granuloma is known to become more likely when fillers injected in an inappropriate area or in a shallow level or over excessively or when filler purity is lower or mixed with fine nano particles smaller than 20 micron in diameter for over $30 \%{ }^{2}$

Foreign body granuloma is diagnosed clinically. Still, histopathological opinions are much helpful in identifying injected materials. Extent literature reported that the occurrence of foreign body granuloma is around 6-24 months. ${ }^{5}$ The patient in this case was relatively healthy without foreign body granuloma risk factors such as internal disease, mental disease, pregnancy, interferon treatment, etc. ${ }^{2}$ Considering this fact, it can be noticed that even in a healthy patient, filler injection could lead to granuloma by alien substance reaction. For a treatment of adrenocortical hormones injection in the lesion area is recommended primarily and whold-body adrenocortical hormone medicine and antibiotics are widely used in parallel. Other reported methods than this include blemomycin injection, local application of Imiquimod or tacrolimun ointment, etc. In this present case, local steroid injection in the lesion and antibiotics were used together. But as the patient showed partial recovery, photodynamic therapy was implemented additionally. The photodynamic therapy is a photo-treatment to make photosensitizer, a material reacting to light, be absorbed into a target cell and flash light in a specific wave length to it in order to destroy targeted lesions selectively. The $660 \mathrm{~mm}$ bio-light stimulates cells involving in cut wound treatment and facilitates blood circulation to promote skin re-generation. For the patient herein who developed ulcerative foreign body granuloma after receiving an alien substance injection by an unauthorized practitioner, localized steroid injection in the lesion was conducted along with the localized photodynamic therapy using the $660 \mathrm{~nm}$ bio-light and Chlorophyll-lipoid complex ICLC, VIRTA-Healer ${ }^{T M}$ ] as a photosensitizer for faster recovery.

Recently, filler injection has increased in the area of plastic surgery for its non-invasive and convenient process. However, prior consensus, etc. are often insufficient about possible post-operational side effects such as granuloma regarding foreign substances. In this situation, more detailed explanation will need 
to be provided, and more treatment cases using the photodynamic therapy should be reported along with additional clinical data related to the treatment guidelines.

\section{REFERENCES}

1. Bae JM, Kim MY, Kang H, Park CJ, Park YM, Cho SH, et al. The clinicopathologic study of foreign body granuloma induced by injection of filler. Korean J Dermatol 2007;45:255-61.

2. Kim YJ, Park MY, Kim YC. Facial foreign body granuloma caused by filler (Artecoll(R)) injection. Korean J Dermatol 2008;
46:491-3.

3. Lemperle G, Romano JJ, Busso M. Soft tissue augmentation with artecoll: 10-year history, indications, techniques, and complications. Dermatol Surg 2003;29:573-87.

4. Kim SA, Lee KS, Cho JW. A case of Platelet-Rich Plasma (PRP) treatment for herpes zoster ophthalmicus-like skin necrosis after filler injection. Korean J Dermatol 2010;48:60-4.

5. Lemperle G, Gauthier-Hazan N, Wolters M, Eisemann-Klein M, Zimmermann U, Duffy DM. Foreign body granulomas after all injectable dermal fillers: part 1. Possible causes. Plast Reconstr Surg 2009;123:1842-63. 